\title{
Correction to: Exposure to arsenic in utero is associated with various types of DNA damage and micronuclei in newborns: a birth cohort study
}

Panida Navasumrit ${ }^{1,2}$, Krittinee Chaisatra', Jeerawan Promvijit', Varabhorn Parnlob', Somchamai Waraprasit' Chalida Chompoobut ${ }^{1}$, Ta Thi Binh ${ }^{3}$, Doan Ngoc Hai ${ }^{3}$, Nguyen Duy Bao ${ }^{3}$, Nguyen Khac Hai ${ }^{3}$, Kyoung-Woong Kim, Leona D. Samson ${ }^{5}$, Joseph H. Graziano ${ }^{6}$, Chulabhorn Mahidol ${ }^{1}$ and Mathuros Ruchirawat ${ }^{1,2^{*}}$

\section{Correction to: Environ Health}

https://doi.org/10.1186/s12940-019-0481-7

Following publication of the original article [1], the author reported that incorrect version of Tables 1, 3, 5 and 6 were published.

The corrected Tables are as follows:

The original article has been corrected.

\begin{abstract}
Author details
${ }^{1}$ Laboratories of Environmental Toxicology/Chemical Carcinogenesis, Chulabhorn Research Institute, Laksi, Bangkok 10210, Thailand. ${ }^{2}$ Center of Excellence on Environmental Health and Toxicology, CHE, Ministry of Education, Ratchathewi, Bangkok 10400, Thailand. ${ }^{3}$ National Institute of Occupational and Environmental Health, Hanoi, Vietnam. ${ }^{4}$ International Environmental Research Center, Gwangju Institute of Science and Technology, Gwangju, South Korea. ${ }^{5}$ Center for Environmental Health Sciences, Massachusetts Institute of Technology, Cambridge, USA.

${ }^{6}$ Department of Environmental Health Sciences, Columbia University, New York, USA.
\end{abstract}

Published online: 24 July 2019

\section{Reference}

1. Navasumrit, et al. Environ Health. 2019;18:51.

\footnotetext{
*Correspondence: mathuros@cri.or.th

'Laboratories of Environmental Toxicology/Chemical Carcinogenesis,

Chulabhorn Research Institute, Laksi, Bangkok 10210, Thailand

${ }^{2}$ Center of Excellence on Environmental Health and Toxicology, CHE, Ministry

of Education, Ratchathewi, Bangkok 10400, Thailand

Full list of author information is available at the end of the article
}

(C) The Author(s). 2019 Open Access This article is distributed under the terms of the Creative Commons Attribution 4.0 International License (http://creativecommons.org/licenses/by/4.0/), which permits unrestricted use, distribution, and reproduction in any medium, provided you give appropriate credit to the original author(s) and the source, provide a link to the Creative Commons license, and indicate if changes were made. The Creative Commons Public Domain Dedication waiver (http://creativecommons.org/publicdomain/zero/1.0/) applies to the data made available in this article, unless otherwise stated. 
Table 1 Demographic characteristics of mothers and infant birth outcomes in Vietnamese pregnancy cohort

\begin{tabular}{|c|c|c|c|c|c|}
\hline \multirow[t]{3}{*}{ Variables } & \multirow{3}{*}{$\begin{array}{l}\text { All } \\
(n=205)\end{array}$} & \multicolumn{3}{|c|}{ Maternal arsenic exposure by toenail As } & \multirow{3}{*}{$p$-value $e^{a}$} \\
\hline & & Low $(<0.5 \mu \mathrm{g} / \mathrm{g})$ & Medium $(0.5-1 \mu \mathrm{g} / \mathrm{g})$ & High $(>1 \mu \mathrm{g} / \mathrm{g})$ & \\
\hline & & $(n=82)$ & $(n=57)$ & $(n=66)$ & \\
\hline Pregnancy BMl $\left(\mathrm{kg} / \mathrm{m}^{2}\right)($ mean $\pm \mathrm{SD})$ & $21.7 \pm 2.2$ & $21.5 \pm 2.2$ & $21.5 \pm 2.3$ & $22.2 \pm 2.1$ & 0.064 \\
\hline Maternal age (years) $($ mean $\pm S D)$ & $26.6 \pm 4.1$ & $27.1 \pm 4.3$ & $25.7 \pm 3.4$ & $26.8 \pm 4.3$ & 0.184 \\
\hline Residential area (As in drinking water) $[\mathrm{n}(\%)]^{\mathrm{b}}$ & & & & & 0.000 \\
\hline Ba Sao $(0.64 \mu \mathrm{g} / \mathrm{L})$ & $31(15.1)$ & $18(22.0)$ & $10(17.5)$ & $3(4.5)$ & \\
\hline Kha Phong $(0.60 \mu \mathrm{g} / \mathrm{L})$ & $3(1.5)$ & $1(1.2)$ & 0 & $2(3.0)$ & \\
\hline Thi Son $(1.64 \mu \mathrm{g} / \mathrm{L})$ & $66(32.2)$ & $48(58.5)$ & $10(17.5)$ & $8(12.1)$ & \\
\hline Hoang Tay $(65.7 \mu \mathrm{g} / \mathrm{L})$ & $30(14.6)$ & $6(7.3)$ & $11(19.3)$ & $13(19.7)$ & \\
\hline Nhant Tan $(61.7 \mu \mathrm{g} / \mathrm{L})$ & $67(32.7)$ & $4(4.9)$ & $23(40.4)$ & $40(60.6)$ & \\
\hline $\operatorname{Van} X a(27.2 \mu \mathrm{g} / \mathrm{L})$ & $8(3.9)$ & $5(6.1)$ & $3(5.3)$ & 0 & \\
\hline Education level [n (\%)] & & & & & 0.019 \\
\hline Elementary school & $15(7.3)$ & $2(2.4)$ & $3(5.3)$ & $10(15.2)$ & \\
\hline Secondary school & $135(65.9)$ & $51(62.2)$ & $44(77.2)$ & $40(60.6)$ & \\
\hline Diploma & $40(19.5)$ & $20(24.4)$ & $7(12.3)$ & $13(19.7)$ & \\
\hline College graduate & $15(7.3)$ & $9(11.0)$ & $3(5.3)$ & $3(4.5)$ & \\
\hline Maternal occupation [n (\%)] & & & & & 0.074 \\
\hline Housewife & $32(15.6)$ & $10(12.2)$ & $9(15.8)$ & $13(19.7)$ & \\
\hline Agricultural worker & $91(44.4)$ & $31(37.8)$ & $26(45.6)$ & $34(51.5)$ & \\
\hline Factory worker & $44(21.5)$ & $18(22.0)$ & $11(19.3)$ & $15(22.7)$ & \\
\hline Employee & $30(14.6)$ & $18(22.0)$ & $10(17.5)$ & $2(3.0)$ & \\
\hline Vendor & $8(3.9)$ & $5(6.1)$ & $1(1.8)$ & $2(3.0)$ & \\
\hline Parity [n (\%)] & & & & & 0.477 \\
\hline 1 person & $75(36.6)$ & $32(39.0)$ & $21(36.8)$ & $22(33.3)$ & \\
\hline 2 persons & $100(48.8)$ & $41(50.0)$ & $30(52.6)$ & $29(43.9)$ & \\
\hline 3 persons & $25(12.2)$ & $8(9.8)$ & $5(8.8)$ & $12(18.2)$ & \\
\hline$\geq 4$ persons & $5(2.4)$ & $1(1.2)$ & $1(1.8)$ & $3(4.5)$ & \\
\hline Antenatal care services [n (\%)] & & & & & 0.464 \\
\hline Province clinic hospital & $20(9.8)$ & $9(11.0)$ & $7(12.3)$ & $4(6.1)$ & \\
\hline District clinic hospital & $23(11.2)$ & $11(13.4)$ & $8(14.0)$ & $4(6.1)$ & \\
\hline Health center of commune & $21(10.2)$ & $9(11.0)$ & $7(12.3)$ & $5(7.6)$ & \\
\hline Private medical center & $125(61.0)$ & $48(58.5)$ & $32(56.1)$ & $45(68.2)$ & \\
\hline Not having antenatal care & $16(7.8)$ & $5(6.1)$ & $3(5.3)$ & $8(12.1)$ & \\
\hline Complications during pregnancy [n (\%)] & & & & & 0.683 \\
\hline No & $189(92.2)$ & $74(90.2)$ & $53(93.0)$ & $62(93.9)$ & \\
\hline Yes & $16(7.8)$ & $8(9.8)$ & $4(7.0)$ & $4(6.0)$ & \\
\hline History of miscarriage [n (\%)] & & & & & 0.409 \\
\hline Never & $175(85.4)$ & $71(86.6)$ & $52(91.2)$ & $52(78.8)$ & \\
\hline 1 time & $23(11.2)$ & $7(8.5)$ & $5(8.8)$ & $11(16.7)$ & \\
\hline 2 times & $4(2.0)$ & $2(2.4)$ & 0 & $2(3.0)$ & \\
\hline$\geq 3$ times & $3(1.5)$ & $2(2.4)$ & 0 & $1(1.5)$ & \\
\hline \multicolumn{6}{|l|}{ Urinary Cotinine ( $\mathrm{g} / \mathrm{mmol}$ creatinine) } \\
\hline Median (range) & nd (nd-8.03) & nd & nd (nd-8.03) & nd & \\
\hline \multicolumn{6}{|l|}{ Infant birth outcomes } \\
\hline Gender & & & & & 0.220 \\
\hline Male $[n(\%)]$ & $109(53.7)$ & $45(54.9)$ & $30(53.6)$ & $34(52.3)$ & \\
\hline Female $[n(\%)]$ & $94(46.3)$ & $37(45.1)$ & $26(46.4)$ & $31(47.7)$ & \\
\hline Birth length $(\mathrm{cm})$ (mean $\pm \mathrm{SD})$ & $49.8 \pm 2.2$ & $50.4 \pm 2.0$ & $49.1 \pm 2.8$ & $49.8 \pm 1.4$ & 0.001 \\
\hline Birth weight $(\mathrm{kg})($ mean $\pm \mathrm{SD})$ & $3.1 \pm 0.8$ & $3.2 \pm 0.8$ & $3.0 \pm 0.8$ & $3.0 \pm 0.8$ & 0.154 \\
\hline Head circumference $(\mathrm{cm})($ mean $\pm S D)$ & $32.2 \pm 2.7$ & $31.9 \pm 2.1$ & $32.6 \pm 4.0$ & $32.2 \pm 1.8$ & 0.336 \\
\hline
\end{tabular}




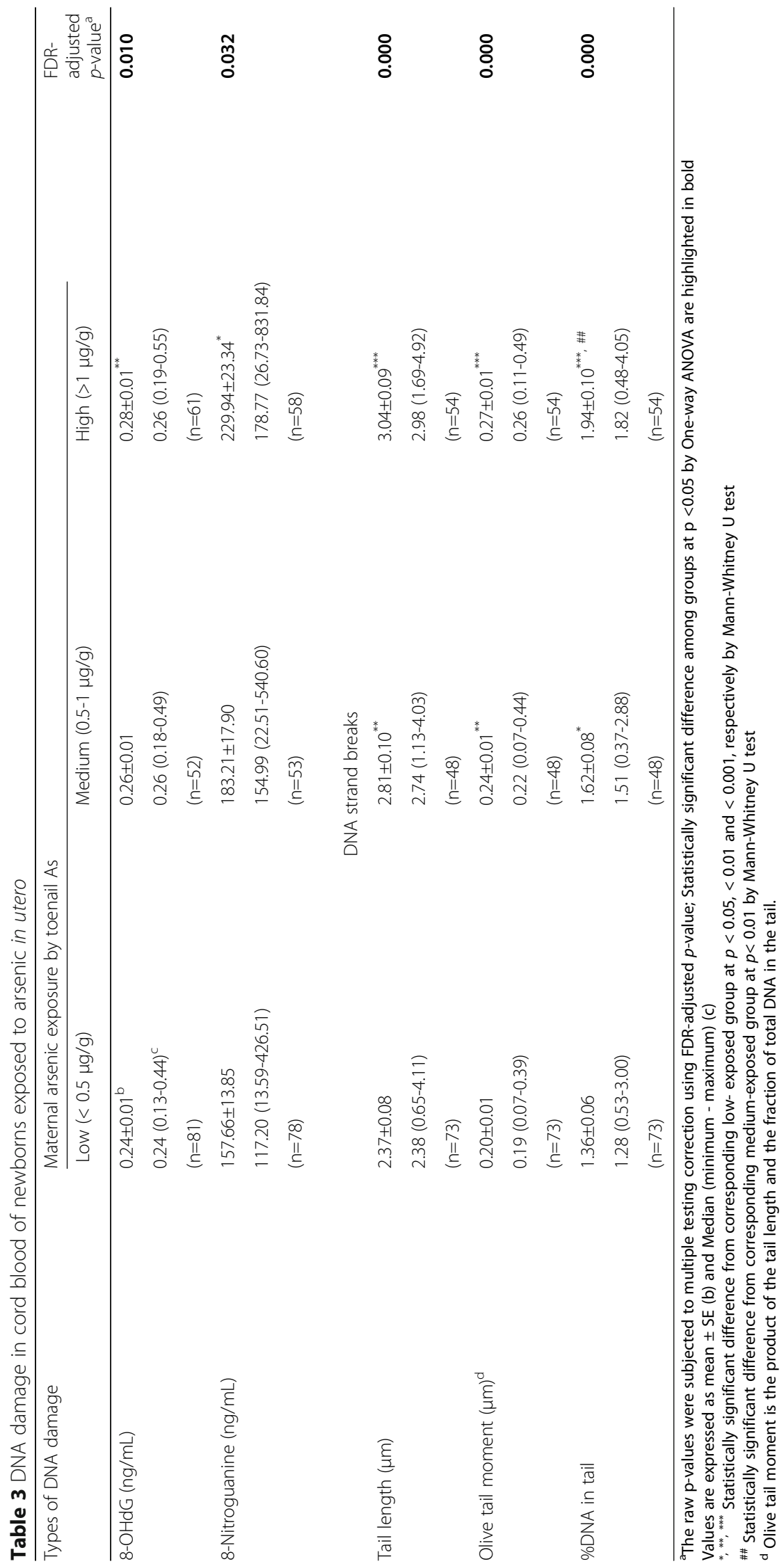




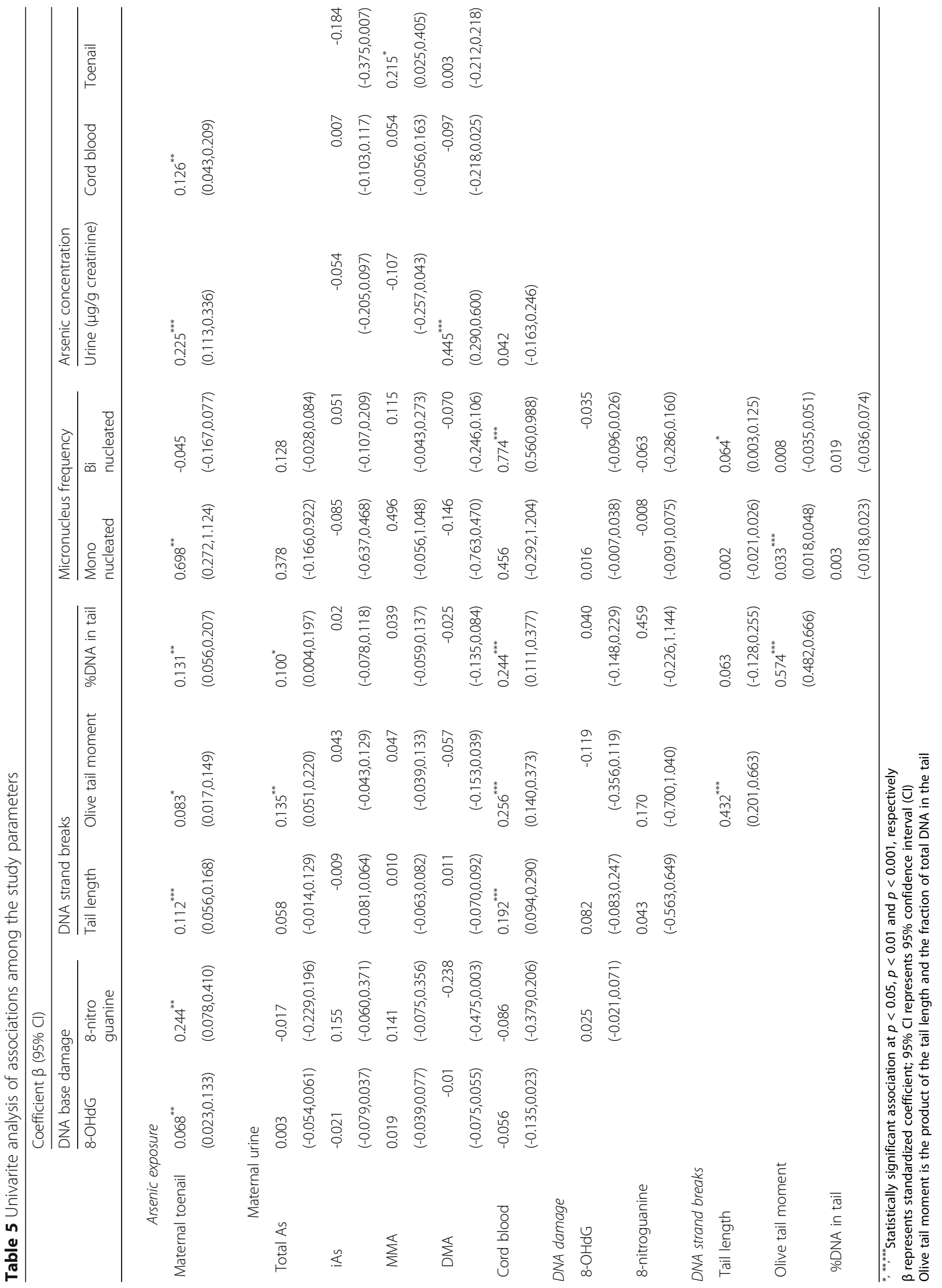


Table 6 Multivariate regression analyses between arsenic exposure and early genotoxic effects in newborns

\begin{tabular}{|c|c|c|c|c|c|c|c|}
\hline & \multicolumn{7}{|c|}{ Coefficient $\beta$ [adjusted p-value] (95\%Cl) } \\
\hline & \multicolumn{2}{|c|}{ DNA base damage } & \multicolumn{3}{|c|}{ DNA strand breaks } & \multicolumn{2}{|c|}{ Micronucleus frequency } \\
\hline & $8-\mathrm{OHdG}$ & 8-Nitroguanine & Tail Length & Olive Mom. & $\% D N A$ in tail & Mononucleated & Binucleated \\
\hline Toenail As & $0.234[0.010]$ & $0.210[\mathbf{0 . 0 3 1}]$ & $0.360[0.000]$ & $0.192[0.000]$ & $0.273[0.000]$ & $0.325[0.000]$ & $-0.029[0.958]$ \\
\hline$(\mu \mathrm{g} / \mathrm{L})$ & $(0.089,0.379)$ & $(0.064,0.356)$ & $(0.221,0.499)$ & $(0.045,0.339)$ & $(0.124,0.422)$ & $(0.177,0.472)$ & $(-0.175,0.118)$ \\
\hline Urinary Total As & $0.003[0.974]$ & $-0.063[0.518]$ & $0.142[0.140]$ & $0.232[\mathbf{0 . 0 0 0}]$ & $0.165[0.073]$ & $0.100[0.220]$ & $0.090[0.146]$ \\
\hline ( $\mu \mathrm{g} / \mathrm{g}$ creatinine) & $(-0.153,0.158)$ & $(-0.219,0.094)$ & $(-0.001,0.285)$ & $(0.089,0.376)$ & $(0.020,0.310)$ & $(-0.056,0.256)$ & $(-0.052,0.232)$ \\
\hline Urinary iAs & $-0.038[0.974]$ & $0.109[0.423]$ & $0.021[0.781]$ & $0.093[0.112]$ & $0.060[0.359]$ & $-0.034[0.339]$ & $0.050[0.146]$ \\
\hline$(\mu \mathrm{g} / \mathrm{L})$ & $(-0.183,0.107)$ & $(-0.037,0.255)$ & $(-0.114,0.156)$ & $(-0.042,0.228)$ & $(-0.077,0.197)$ & $(-0.213,0.144)$ & $(-0.119,0.219)$ \\
\hline Urinary MMA & $0.012[0.974]$ & $0.076[0.518]$ & $0.054[0.538]$ & 0.087 [0.027] & $0.073[0.268]$ & $0.163[0.091]$ & $0.153[0.114]$ \\
\hline$(\mu \mathrm{g} / \mathrm{L})$ & $(-0.137,0.161)$ & $(-0.074,0.226)$ & $(-0.085,0.192)$ & $(-0.052,0.226)$ & $(-0.068,0.214)$ & $(0.016,0.311)$ & $(0.011,0.295)$ \\
\hline Urinary DMA & $-0.006[0.974]$ & $-0.060[0.518]$ & $0.085[0.538]$ & $0.003[0.056]$ & $0.029[0.375]$ & $-0.012[0.255]$ & $-0.011[0.227]$ \\
\hline$(\mu \mathrm{g} / \mathrm{L})$ & $(-0.155,0.143)$ & $(-0.209,-0.090)$ & $(-0.053,0.223)$ & $(-0.148,0.153)$ & $(-0.123,0.181)$ & $(-0.208,0.183)$ & $(-0.199,0.177)$ \\
\hline Cord blood & $-0.105[0.503]$ & $-0.026[0.732]$ & $0.270[0.001]$ & $0.316[\mathbf{0 . 0 0 0}]$ & $0.264[\mathbf{0 . 0 0 1}]$ & $0.094[0.255]$ & $0.519[\mathbf{0 . 0 0 0}]$ \\
\hline$(\mu \mathrm{g} / \mathrm{L})$ & $(-0.254,0.044)$ & $(-0.177,0.125)$ & $(0.131,0.409)$ & $(0.178,0.454)$ & $(0.125,0.404)$ & $(-0.054,0.242)$ & $(0.380,0.657)$ \\
\hline
\end{tabular}

Model was adjusted for the covariates including age, BMI, education, occupation, gestational age of sample collection during pregnancy (urine and nail samples) and baby delivery (cord blood sample)

The raw $\mathrm{p}$-values were subjected to multiple testing corrections using FDR-adjusted $p$-value and the statistically significant are highlighted in bold

$\beta$ represents standardized coefficient; $95 \% \mathrm{Cl}$ represents $95 \%$ confidence interval (Cl)

Olive tail moment is the product of the tail length and the fraction of total DNA in the tail 